\title{
Personalbemessung im Jugendamt
}

\section{Bayerisches Pilotprojekt entwickelt geeignete Instrumente}

\author{
Irmgard Schroll-Decker, Jörg Strobel, André Schilay
}

Mehr Personal bedeutet nicht unbedingt bessere Aufgabenerfüllung. Aber welche Standards und Methoden der Feststellung des Personalbedarfs sind beispielsweise in Jugendämtern fachlich richtig? Eine Untersuchung in Bayern versuchte, angemessene Antworten zugeben.

Gesellschaftliche Herausforderungen wie Vernachlässigung und Misshandlung von Kindern veranlassen häufig, die sozialen Dienste der Jugendämter zu hinterfragen. Neue Aufgaben wie der Schutzauftrag bei Kindeswohlgefährdung ( $\$ 8$ a SGB VIII) werden nach dem Maximalprinzip implementiert und den Mitarbeitern zusätzlich aufgebürdet. Sobald eine neue Hilfeform im Gesetz verankert ist, flaut das öffentliche Interesse ab.

Prof. Dr. Irmgard Schroll-Decker lehrt an der Hochschule Regensburg

Sozialmanagement und Bildungsarbeit. E-Mail Irmgard.Schroll-Decker@ hs-regensburg.de

Jörg Strobel ist Diplom-Sozialpädagoge und Wirtschaftsfachwirt. Er arbeitete acht Jahre für den öffentlichen Dienst der Bundeswehr im Ressort Personalmanagement. Seit fünf Jahren ist er mit benachteiligten Kindern, Jugendlichen und Erwachsenen in unterschiedlichen Arbeitsfeldern der Jugendhilfe und Erwachsenenbildung tätig.

E-Mail Joerg Strobel

strobel.joerg@googlemail.com

André Schilay ist Diplom-

Sozialpädagoge und Master of Social Management. Seit 2001 ist er angestellt beim Kreisjugendamt Neumarkt, wo er seit 2009 in der Stabsstelle

»Koordinierende Kinderschutzstelle -

Netzwerk frühe Kindheit« tätig ist.

E-Mail schilay.andre@

landkreis-neumarkt.de
Den sozialen Dienst vorzuhalten und ihn zu erbringen, ist Sache der Personalsteuerung der Behörde. Ausgelöst vom Beispiel der verpflichtenden Regelung von Verantwortungsbereichen im Kontext des $₫ 8$ a SGB VIII fand in der Fachpraxis eine enorme Sensibilisierung statt (vgl. Haller, 2008, 11). Parallel zur Aufgabenmehrung wurde für die Bearbeitung mehr Personal gefordert. Nun entstand die Frage, ob mit dieser Gleichung bereits eine intelligente Aufgabenerfüllung gewährleistet ist.

Das Bayerische Landesjugendamt nahm dies zum Anlass, in Kooperation mit dem Bayerischen Landkreistag und dem Institut für Sozialplanung und Organisationsentwicklung gemeinsam mit drei Jugendämtern das Pilotprojekt »Personalbemessung der Jugendämter in Bayern $(\mathrm{PeB})$ « aufzusetzen und sich des Instruments der Personalbedarfsfeststellung zu bedienen. (1)

\section{Fallzahlen und Arbeitsprozesse}

\section{Kernprozesse}

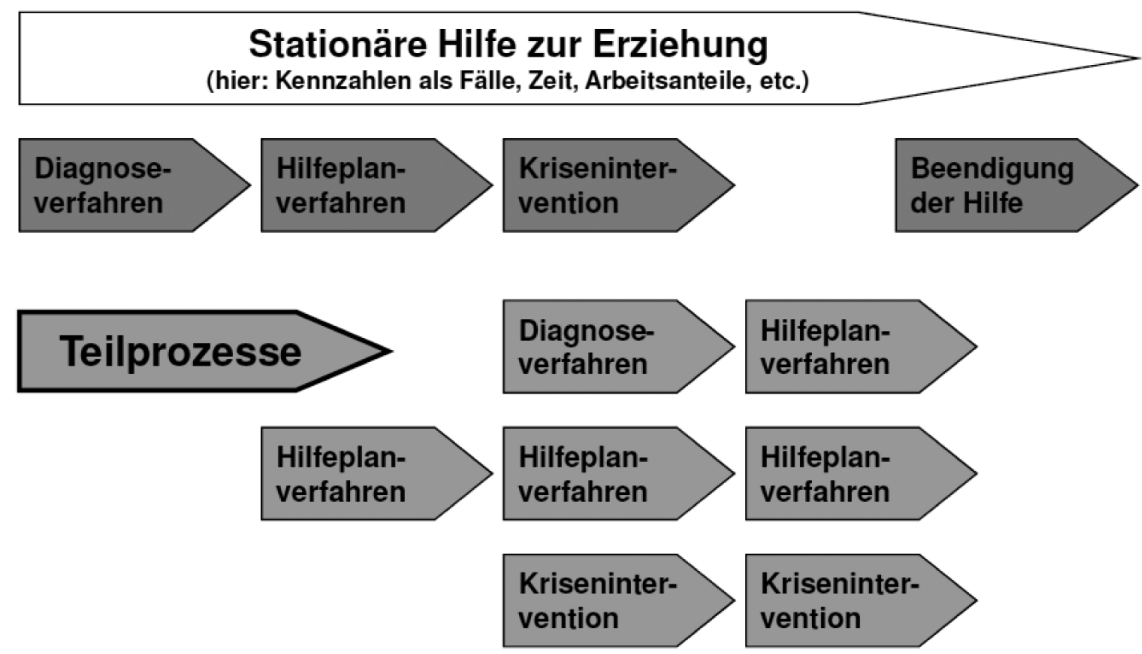

Beendigung der Hilfe

Abb. 1: Arbeitsprozesse am Beispiel stationäre Hilfe zur Erziehung

Quelle: Szlapka, 2008, 74, modifiziert durch J. S. 


\section{Bezugsgrößen der Personalbemessung}

Die Personalbemessung arbeitet mit drei Variablen: der Jahresarbeitszeit, den Fallzahlen sowie den Kern- und Teilprozessen. Die Jahresarbeitszeit einer Vollzeitäquivalenzstelle beträgt 1.583 Stunden. (2)

Die Fallzahlen ermitteln sich aus den tatsächlich registrierten Fällen einer Hilfeleistung. In ihnen spiegeln sich die Einwohnerwerte (Gesamtanzahl eines Bezirkes und der Anteil junger Menschen) und die Anhaltszahlen (Anzahl der Leistungsfälle pro Fachkraft) wider.

Inhaltlich sind bei den einzelnen Diensten Kern- und Teilprozesse sowie einzelne Tätigkeiten zu bestimmen. $\mathrm{Zu}$ berükksichtigen sind zudem die mittleren Bearbeitungszeiten und die Anhaltswerte (Pauschalwerte) aus interkommunalen Vergleichswerten (vgl. Szlapka, 2008, 77).

\section{Vorgehen bei der Personalbemessung}

Die Berechnung beginnt mit den Arbeitsprozessen, die innerhalb der Dienstleistung (z. B. Inobhutnahme) definiert werden. Eine Tätigkeit wie beispielsweise die Informationsaufnahme kann fachlich unabhängig vom jeweiligen Fall standar- disiert werden. Die durchschnittliche Arbeitsmenge ist an die Tätigkeiten und nicht an die Fälle geknüpft. Die jeweilige Hilfeleistung legt mittels der fachlichen Standards den erforderlichen Ressourceneinsatz fest (vgl. Szlapka, 2008, S. 73 ff.).

\section{Arbeitszeitkomponenten für die Personalbemessung}

Die Jahresarbeitszeit von 1.563 Stunden besteht aus der Ausführungszeit einschließlich eines kleinen Anteils ( 1 bis 5

\section{»Viele Tätigkeiten - beispielsweise die Informationsaufnabme - können unabhängig vom jeweiligen Fall standardisiert werden"}

Die Leistung »Stationäre Hilfe zur Erziehung « umfasst beispielsweise die Arbeitsschritte Erstkontakt, Diagnose, Hilfeplanverfahren, bei Bedarf Krisenintervention und Beendigung der Hilfe (vgl. Abb. 1). Teilprozesse können aus einer Reihe von Tätigkeiten bestehen (Einladung zum Hilfeplangespräch, Einholen von Stellungnahmen u. a. m.), die geplant werden müssen oder als Sofortarbeit anfallen. Fachexperten im Jugendamt müssen Tätigkeiten fallweise an spezifische Anforderungen anpassen oder flexibel reagieren, wenn sich Stellgrößen (z. B. eine häufigere Überprüfung) verändern, die zu einem Anstieg von Tätigkeiten ( $\mathrm{z}$. B. Elternkontakte) führen können (vgl. Szlapka, 2008, 74).

\section{Differenzierung der Arbeitszeit}
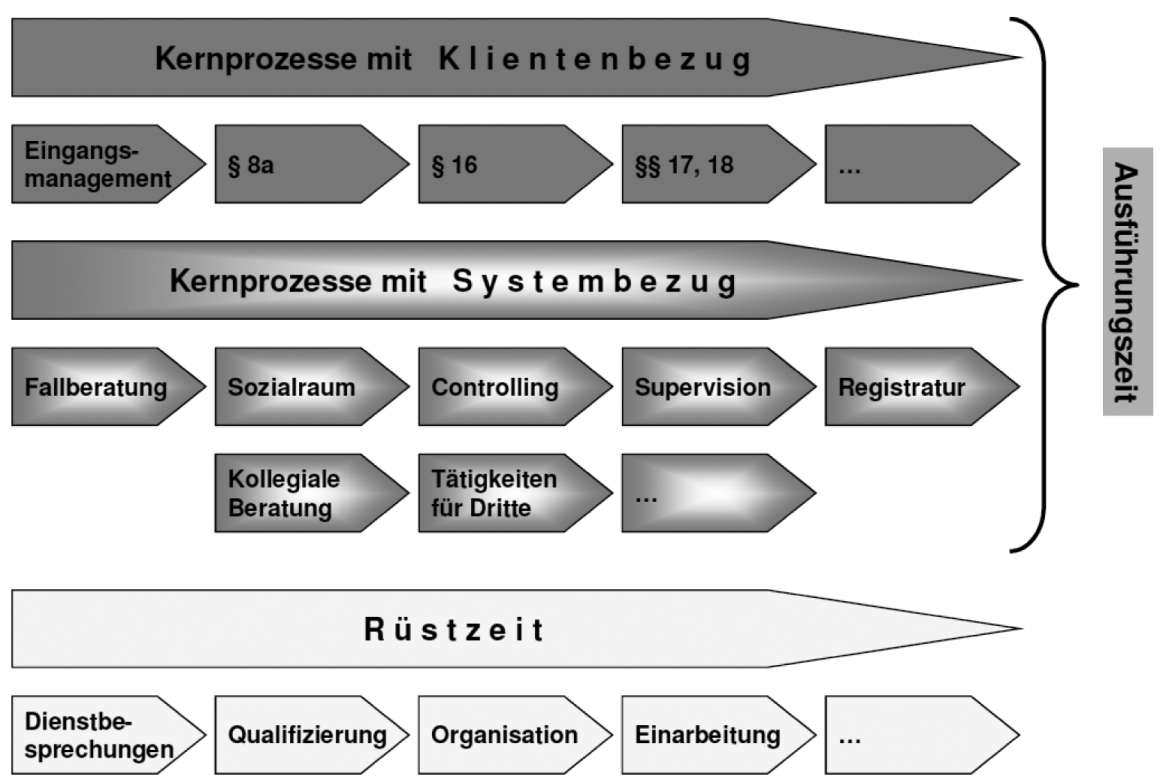

Abb. 2: Bausteine der Arbeitszeit

Quelle: Quelle: Fink et al., 2009, 7, modifiziert durch J. S.

Prozentpunkte) an Verteilzeit für unvorhersehbare Arbeitsprozesse und der sogenannten Rüstzeit zur Vor- und Nachbereitung (vgl. Szlapka, 2008, 79 f.).

Den Ausgangspunkt für die Ermittlung der Zeitangaben für die Kernprozesse bilden die fachlichen Standards (vgl. Abb. 2). Danach bemisst sich die Ausführungszeit nach Klienten- und nach Systembezug. So werden Aufwendungen bestimmt, die unmittelbar und mittelbar Auswirkungen auf die Klientel haben. Die Rüstzeit dagegen beinhaltet Zeitanteile, die nicht direkt in die Kernprozesse einfließen (vgl. Szlapka, 2008, 80 ff.).

Qualitative Standards und finanzielle Ressourcen bedingen sich wechselseitig: Ist es fachlicher Konsens, pro Quartal von einem auf zwei Elterngespräche zu wechseln, können die personellen Auswirkungen unmittelbar abgeleitet werden. Das Jugendamt kann transparent darlegen, wo ein Defizit an Ressourcen besteht und welche Folgen es für die Leistungserbringung hat. Die Personalbedarfsbestimmung fokussiert nicht den Personalstamm, sondern liefert eine differenzierte Betrachtungsweise über benötigte und gewünschte Qualitäten (vgl. Szlapka, 2008, 87).

\section{Fragmente einer Umsetzung der Personalbemessung}

Die folgenden Ausführungen beziehen sich auf das konkrete Vorgehen eines beteiligten Jugendamtes.

- Erstkontakt: Für den Kernprozess Erstkontakt wurde mit allen Fachkräften unter Beachtung der gesetzlichen Bestimmungen ein Ablaufplan erarbeitet. Bei der ersten Kontaktaufnahme klärt 
sich der Bedarf an Jugendhilfeleistungen und eine eventuelle Weitervermittlung. Werden Leistungen der Jugendhilfe zugesprochen, folgt eine weitere Sachverhaltsklärung (z. B. eine sozialpädagogische Diagnose), bevor an die leistungsverantwortliche Fachkraft verwiesen wird. Kollegiale Beratung kann hier in Anspruch genommen werden. Das Resultat ist einheitliches Arbeitskonzept inklusive Sonderfallbehandlungen. Auch die Teilprozesse wurden gemäß dem definierten Kernprozess charakterisiert. Alle Teilprozesse sind nach einem vereinheitlichten Muster beschrieben und an das örtliche Jugendamt angepasst.
- Auftragszeitbestimmung: Zur Auftragszeitbestimmung werden die Teilprozesse in einzelne Aktivitäten unterschieden und mit Zeitangaben unterlegt. Exakte Aussagen wurden zu den Beratungsgesprächen, den Fahrtaufwendungen, zur kollegialen Beratung und Reflexion usw. getroffen.

- Systembezug: Tätigkeiten mit Systembezug (z. B. Fortbildung, Netzwerkarbeit) sind eigens ermittelt und zeitlich beziffert. Auf der Basis eines Arbeitsjahres sind auch die durchschnittliche Rüstzeit (individuelle Vor- und Nachbereitungszeiten und dienstliche Belange wie Dienstbesprechungen) vereinbart.

\section{Kernprozess: Eingangsmanagement}

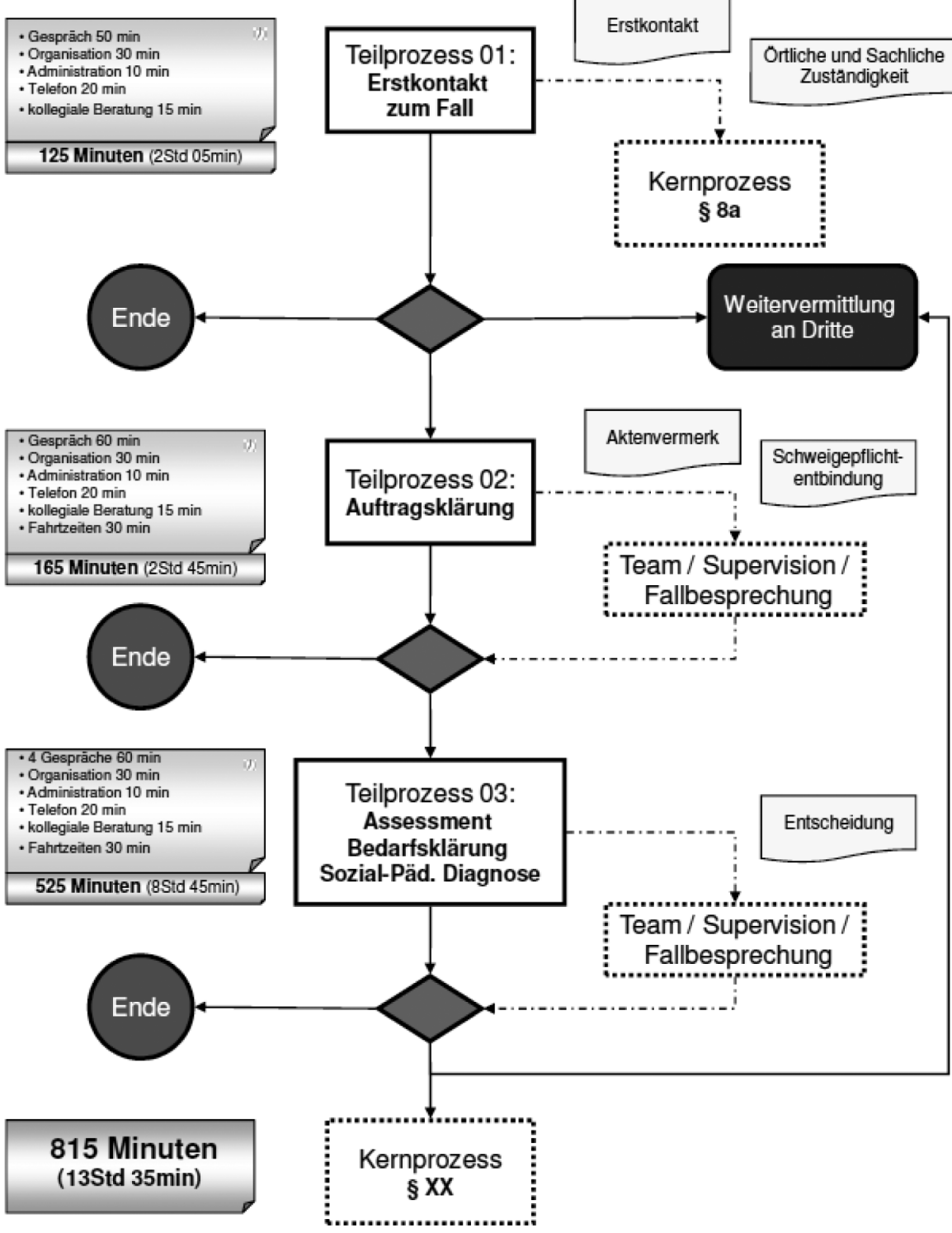

Abb. 3: Endprodukt (einschließlich Zeitangaben) zum Prozess Eingangsmanagement Quelle: INSO, 2008/2009, modifiziert durch J. S.

Verbindlich definiert und vereinbart sind folgende Aktivitäten:

- Beratungsgespräch: Gespräche zwischen Fachkräften und Eltern und mit den noch nicht Volljährigen, Unterredungen mit Dritten (Schule, andere Fachkräfte etc.).

- Kurzgespräch: telefonische und kurze persönliche Gespräche zur Abstimmung, Vereinbarung oder Erörterung von bündigen Sachverhalten.

- Dokumentation: Zusammenstellen von Dokumenten und Aktenführung (Aktennotizen, Hilfeplanprotokolle, Stellungnahmen, Gutachten).

- Administration: Tätigkeiten wie Kopieren von Unterlagen, Schreiben und Versenden von Einladungen oder Antragstellung für Dienstreisen.

- Kollegiale Beratung und Reflexion: Zusammenwirken mehrerer Fachkräfte bei der Entscheidung über die Auswahl der Hilfeart und des Umfangs.

- Verhandlung und Erörterung: Teilnahmen von Fachkräften an Erörterungen oder Verhandlungen.

- Fahrtzeiten: Weg- und Fahrtzeiten ohne generelle Zeitwerte in den Teilprozessen (z. B. zusätzliche Fahrzeiten, die zu Ausführungszeiten addiert werden).

Der Kernprozess Eingangsmanagement besteht aus einem geschätzten Gesamtaufwand von 815 Minuten, die sich aus 125 Minuten Aufwand im ersten, 165 Minuten im zweiten und 525 Minuten im dritten Teilprozess zusammensetzen (vgl. Abb. 3). Die Zeitanteile steigen mit der Konkretisierung des Falls. Erfasst wurde die Zeit ab dem Zugang bis zur ersten Entscheidung. Sie erstrecken sich auf maximal eine Arbeitswoche. Bis zum Teilprozess »03 (Assessment) « können zwei bis drei Wochen beansprucht werden.

\section{Personalbedarfsberechnung}

Die mittleren Bearbeitungszeiten und ihre Verteilung auf Kern- und Rüstzeiten werden nun mit den tatsächlichen Fallzahlen eines Jahres in Verbindung gebracht.

Beispiel: $\quad$ Anzahl der Fälle im Jahr X Kernprozess Falleingang: 506 Fälle

Kernprozess $\int 8$ :

123 Fälle

Kernprozess $\int 16$ :

338 Fälle 


\begin{tabular}{|c|c|c|c|c|c|c|c|}
\hline \multirow{2}{*}{\multicolumn{4}{|c|}{ Berechnungsgrundlagen }} & \multicolumn{4}{|c|}{ Personalbemessungsbereiche } \\
\hline & & & & \multirow[t]{2}{*}{$\begin{array}{l}\text { Algemeiner } \\
\text { Sozialdienst }\end{array}$} & \multirow[t]{3}{*}{ Adoptionswesen } & \multirow{2}{*}{$\begin{array}{l}\text { Jugandgo- } \\
\text { richtshilfe }\end{array}$} & \multirow[t]{3}{*}{$\begin{array}{c}\text { Pflegekinder- } \\
\text { wesen }\end{array}$} \\
\hline \multirow{2}{*}{\begin{tabular}{|c} 
Leistungsbreiche \\
KP Falleingang
\end{tabular}} & \multirow[t]{2}{*}{ Zeiten far $\mathrm{KP} / \mathrm{TP}$} & \multicolumn{2}{|c|}{ Fallzahlen Jahr $\mathrm{X}$} & & & & \\
\hline & & & & & & & \\
\hline TP 01a & $30 \mathrm{~min}$ & 253 & $50 \%$ & 126,50 Std & & & \\
\hline TP $01 \mathrm{~b}$ & $125 \mathrm{~min}$ & 253 & $50 \%$ & $527,08 \mathrm{Std}$ & & & \\
\hline TP 02 & $165 \mathrm{~min}$ & 127 & $25 \%$ & $349,25 \mathrm{Std}$ & & & \\
\hline TP 03 & $525 \mathrm{~min}$ & 102 & $20 \%$ & $892,50 \mathrm{Std}$ & & & \\
\hline \multicolumn{5}{|l|}{ KP \& sa } & & & \\
\hline TP 01 & $75 \mathrm{~min}$ & 160 & $100 \%$ & 200,00 Std & & & \\
\hline TP 02 & $315 \min$ & 123 & $70 \%$ & $645,75 \mathrm{Std}$ & & & \\
\hline TP 03 & $240 \min$ & 32 & $25 \%$ & 128,00 Std & & & \\
\hline \multicolumn{5}{|l|}{ KP \& 16-1 } & & & \\
\hline TP 01 & $244 \min$ & 253 & $75 \%$ & $1.028,87$ Std & & & \\
\hline \multicolumn{5}{|l|}{ KP \& 16-2 } & & & \\
\hline TP 01 & 707 min & 85 & $25 \%$ & $1.001,58$ Std & & & \\
\hline \multicolumn{5}{|l|}{ KP Ş 17,18 } & & & \\
\hline TP 01 & $398 \mathrm{~min}$ & 170 & $100 \%$ & $1.127,67 \mathrm{Std}$ & & & \\
\hline TP 02 & $85 \mathrm{~min}$ & 34 & $20 \%$ & 48,17 Std & & & \\
\hline \multicolumn{5}{|l|}{ KP s 27 ambulant } & & & \\
\hline Summe der TP 1-7 & $1.652 \min$ & 99 & $100 \%$ & $2.725,80 \mathrm{Std}$ & & & \\
\hline \multicolumn{5}{|l|}{ KP 乌 27 teilstationär } & & & \\
\hline Summe der TP $1-7$ & $1.652 \mathrm{~min}$ & 41 & $100 \%$ & $1.128,87 \mathrm{Std}$ & & & \\
\hline \multicolumn{5}{|l|}{ KP 5 27 stationâr } & & & \\
\hline Summe der TP 1-7 & & 72 & $100 \%$ & $1.982,40 \mathrm{Std}$ & & & \\
\hline \multicolumn{4}{|l|}{ KP \& 33} & & & & \\
\hline \multirow{2}{*}{\multicolumn{4}{|c|}{$\begin{array}{l}\text { Summe der TP 1-3 } \\
\text { KP \& } 33 \text { Oberprüfung }\end{array}$}} & & & & 759,50 Std \\
\hline & & & & & & & \\
\hline TP 04 & $540 \mathrm{~min}$ & 9 & $100 \%$ & & & & 81,00 Std \\
\hline TP O4a & $2.880 \mathrm{~min}$ & 1 & $100 \%$ & & & & $48,00 \mathrm{Std}$ \\
\hline \multirow{2}{*}{\multicolumn{5}{|c|}{$\begin{array}{l}\text { TP } 04 b \\
\text { KP s } 42\end{array}$}} & & & 594,00 Std \\
\hline & & & & & & & \\
\hline \multirow{2}{*}{\multicolumn{5}{|c|}{$\begin{array}{l}\text { Summe TP 1-2 } \\
\text { KP \& } 50\end{array}$}} & & & \\
\hline & & & & & & & \\
\hline TP 1-2 & $480 \mathrm{~min}$ & 254 & $80 \%$ & $2.032,00 \mathrm{Std}$ & & & \\
\hline TP 03 & $230 \mathrm{~min}$ & 63 & $20 \%$ & $241,50 \mathrm{Std}$ & & & \\
\hline TP 04 & $60 \mathrm{~min}$ & 50 & $20 \%$ & $50,00 \mathrm{Std}$ & & & \\
\hline \multicolumn{4}{|l|}{ KP \& 51} & & & & \\
\hline keine Berechnung mgl. & $0 \mathrm{~min}$ & 0 & $0 \%$ & & 00,00 Std & & \\
\hline \multicolumn{4}{|l|}{ KP \$ 52} & & & & \\
\hline TP 01 & $12 \min$ & 572 & $100 \%$ & & & 114,40 Std & \\
\hline TP 02 & $280 \mathrm{~min}$ & 330 & $100 \%$ & & & $1.540,00$ Std & \\
\hline TP 03 & $53 \mathrm{~min}$ & 19 & $100 \%$ & & & $16,78 \mathrm{Std}$ & \\
\hline Gesamtkliente & nzeit je Ber & eich & & $14.296,93 \mathrm{Std}$ & $00,00 S_{t d}$ & $1.671,18 \mathrm{Std}$ & $1.482,50 \mathrm{Std}$ \\
\hline
\end{tabular}

Abb. 4: Berechnungsschema zur Klientenzeit

Quelle: INSO, 2009, modifiziert durch J. S.

\begin{tabular}{|c|c|c|c|c|c|c|c|c|}
\hline \multirow{2}{*}{\begin{tabular}{|l}
1.583 Jahresarbeitszeitstunden \\
E r f a s s u n g s b e r e i c h e
\end{tabular}} & \multicolumn{8}{|c|}{ Personalbemessungsbreiche } \\
\hline & $\begin{array}{l}\text { Allgemeiner } \\
\text { Sozialdienst }\end{array}$ & \begin{tabular}{|c|} 
Zeitan- \\
teile
\end{tabular} & Adoptionswesen & $\begin{array}{c}\text { Zeitan- } \\
\text { teile }\end{array}$ & $\begin{array}{c}\begin{array}{l}\text { Jugendgerichts } \\
\text { hilfe }\end{array} \\
\text { - }\end{array}$ & 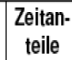 & $\begin{array}{l}\text { Pflegekinder- } \\
\text { wesen }\end{array}$ & \begin{tabular}{|c} 
Zeitan- \\
teile
\end{tabular} \\
\hline Beschăftigungsumfang (VZ̊̊) & $6,25 \mathrm{ST}$ & $100 \%$ & $0,50 \mathrm{ST}$ & $100 \%$ & $0,85 \mathrm{ST}$ & $100 \%$ & $1,00 \mathrm{ST}$ & $100 \%$ \\
\hline Auftagszeit & $9.893,75 \mathrm{Std}$ & $100 \%$ & $791,50 \mathrm{Std}$ & $100 \%$ & $1.345,55 \mathrm{Std}$ & $100 \%$ & $1.583,00 \mathrm{Std}$ & $100 \%$ \\
\hline Rüstzeit & $436,10 \mathrm{Std}$ & $2,59 \%$ & $60,50 \mathrm{Std}$ & $30,17 \%$ & $60,50 \mathrm{Std}$ & $3,08 \%$ & $62,30 \mathrm{Std}$ & $3,26 \%$ \\
\hline Systemzeit & $1.320,00 \mathrm{Std}$ & $7,84 \%$ & 140,00 Std & $69,83 \%$ & 140,00 Std & $7,13 \%$ & $280,00 \mathrm{Std}$ & $14,64 \%$ \\
\hline Klientenzeit & $14.296,93 \mathrm{Std}$ & $84,93 \%$ & $00,00 \mathrm{Std}$ & $0,00 \%$ & $1.671,18 \mathrm{Std}$ & $85,17 \%$ & $1.482,50 \mathrm{Std}$ & $77,50 \%$ \\
\hline Verteilzeit & $780,65 \mathrm{Std}$ & $4,64 \%$ & $00,00 \mathrm{Std}$ & $0,00 \%$ & $90,55 \mathrm{Std}$ & $4,61 \%$ & $88,15 \mathrm{Std}$ & $4,61 \%$ \\
\hline Bedarf an Auftragszeit & $16.833,68 \mathrm{Std}$ & $100 \%$ & 200,50 Std & $100 \%$ & $1.962,23 \mathrm{Std}$ & $100 \%$ & $1.912,95 \mathrm{Std}$ & $100 \%$ \\
\hline Mehr-oder Minderbedarf & $6.939,93 \mathrm{Std}$ & & $-591,00$ Std & & $616,68 \mathrm{Std}$ & & $329,95 \mathrm{Std}$ & \\
\hline Entsprechende VZÄ & $4,38 \mathrm{ST}$ & & $-0,37 \mathrm{ST}$ & & $0,39 \mathrm{ST}$ & & $0,21 \mathrm{ST}$ & \\
\hline
\end{tabular}

Abb. 5: Ergebnismaske zur Personalbedarfsermittlung

Quelle: INSO, 2009, modifiziert durch J. S.
Die Fallzahlen werden in ein prozentuales Verhältnis zum jeweiligen Teilprozess im Kernprozess gesetzt. Im Falleingang mit 506 Fällen ist der erste Teilprozess in zwei Bereiche zu jeweils 50 Prozent aufgeteilt. 253 Fälle wurden angenommen und zugleich abgeschlossen oder weitervermittelt. Dafür wurde ein geringerer Arbeitsaufwand angesetzt. Die restlichen 253 Fälle erforderten eine intensivere Bearbeitung und Entscheidungsfindung. Um die benötigten Zeitanteile zu erhalten, wurden diese mit den mittleren Bearbeitungszeiten im Kernprozess oder Teilprozess multipliziert. Von 253 Fällen wurden im Teilprozess 02 noch 127 Fälle $(25 \%)$ begleitet und im Teilprozess 03 erhielten 20\% (102 Fälle) von 253 Fällen sozialpädagogische Unterstützung. Diese Zeitwerte werden den Personalbemessungsbereichen zugeordnet: Das Eingangsmanagement obliegt dem Allgemeinen Sozialdienst. Aus der Berechnung kann die System- und Rüstzeit ermittelt werden (vgl. Abb. 4).

\section{Resultate und kritische Anmerkungen}

Multipliziert man die Jahresarbeitszeitstunden mit dem Beschäftigungsumfang, so ergibt sich die zur Verfügung stehende Auftragszeit (z. B. 1.583 Std. x 6,25 ST = 9.893,75 Std.). Die Summe der Werte der Klienten-, System-, Rüst- und Verteilzeit ergibt den Bedarf an Auftragszeit. Nun können die Auftragszeit und der Bedarf miteinander verglichen werden. Die Differenz zwischen Ist-Stand und ermitteltem Bedarf zeigt die Über- oder Unterdeckung an. Dividiert man das Ergebnis mit den Jahresarbeitsstunden, so erhält man den Mehr- oder Minderbedarf an Vollzeitäquivalenzstellen (vgl. Abb. 5).

An der Personalbemessung wird kritisiert, dass sich die Mitarbeiter zu Objekten degradiert sehen. Bei einer institutionalisierten Anwendung entsteht der Verdacht, es handle sich um eine Bereinigung des Stellenplans inklusive einer nachhaltigen Reduzierung der Kostenaufwendungen für das Personal (vgl. Krems, 2009, $1)$.

Diese Vorbehalte können jedoch mit Hilfe einer fachlichen Anleitung, einer Beteiligung der Professionsvertreter, einer transparenten Durchführung und einer verständnisvollen Personalführung ent- 
kräftet werden. Besonders relevant ist die Suche nach Lösungen vor Ort, da sie in den meisten Fällen zu besseren Ergebnissen führt als nach dem Top-Down-Prinzip umgesetzte Zwangsmaßnahmen (vgl. Fischer \& Fischer, 2009, S. 40 f.).

Unabhängig von den involvierten Fachkräften spielen das Entlohnungssystem sowie der Arbeitsmarkt eine bedeutsame Rolle. Insbesondere die politischen Entscheidungsträger werden sich angesichts der Transparenz des Verfahrens genau überlegen müssen, welche Qualitätsstandards sie für die sozialen Dienste setzen (wollen). Nachgewiesene Abweichungen bei der Personalbesetzung können dann weniger auf fachliche Unzulänglichkeiten zurückgeführt werden, sondern lassen sich stärker mit dem politischen Willen und den gewährten Ressourcen (z. B. im Kontraktmanagement, bei Zielvereinbarungen) in Relation bringen.

\section{Anmerkungen}

(1) Dokumentiert ist das Projekt im Handbuch und Projektbericht (ZBFS, Projektbericht, 2009).

(2) Bei Mitarbeitern in Teilzeit wird die Jahresarbeitszeit gesondert angepasst (vgl. BMI, 2007, 211).

\section{Literatur}

Bundesministerium des Inneren - BMI (Hg.): Handbuch für Organisationsuntersuchungen und Personalbedarfsermittlung. Berlin 2007.

Fink, T. \& Hastrich, B. \& Szlapka, M.: Personalbemessung der Jugendämter in Bayern (PeB). Qualitative und quantitative Leistungsziele. Ein Modell zur Berechnung des Personalbedarfs in den Bayerischen Jugendämtern. Essen 2009.

Fischer, K. \& Fischer, M.: Der Volkswirt: Köpfe der Wirtschaft. Ordnung ohne Formeln. In: Wirtschaftswoche Nr.43. Düsseldorf 2009, S. 40-41.

Haller, S.: Leistungsgrenzen und Entwicklungsperspektiven des ASD. In: Verein für Kommunalwissenschaften e. V. (Hg.): Konzepte der Personalbemessung und Qualitätsstandards des ASD. Aktuelle Beiträge zur Kinder- und Jugendhilfe 67. Berlin 2008, S. 11-12.

Hutzschenreuter, T.: Allgemeine Betriebswirtschaftslehre. Grundlagen mit zahlreichen Praxisbeispielen. 3. Auflage. Wiesbaden 2009.

Institut für Sozialplanung und Organisationsentwicklung INSO e. V.: Darstellung und Beschreibung der Kernprozesse. Von der Vorlage zur fertigen Ablauforganisation. Essen/Bernried 2008/2009.

Institut für Sozialplanung und Organisationsentwicklung INSO e. V.: Ein Modell zur Berechnung des Personalbedarfs. Essen/Bernried 2009.

Krems, B.: Zur Kritik der analytischen Personalbemessung. Welchen Stellenwert sollte die analytische Personalbemessung im modernen Verwaltungsmanagement haben? Beitrag im Online-Verwaltungslexikon. Köln: 2009. Internet http://www.olev.de/p/persbed-kritik.htm (Zugriff: 27.09.2009).

Sauter, R.: Vor neuen Herausforderungen. Wie helfen, wenn Menschen fehlen. In: Evangelischer Erziehungsverband in Bayern e. V. (Hg.): Erziehungshilfe hilflos wenn Fachkräfte fehlen. Steigender Personalbedarf in der sozialen Arbeit. Ausgabe Nr. 1. Juli 2009. 27. Jahrgang. Nürnberg 2009, S. 10-15.

Szlapka, M.: Qualitative und quantitative Leistungsziele. Ein Modell zur Berechnung des Personalbedarfs in den Sozialen Diensten (nicht nur) in der Stadtgemeinde Bremen. In: Verein für Kommunalwissenschaften e. V. (Hg.): Konzepte der Personalbemessung und Qualitätsstandards des ASD. Aktuelle Beiträge zur Kinder- und Jugendhilfe 67. Berlin 2008, S. 73-88.

Zentrum Bayern Familie und Soziales (ZBFS) - Bayerisches Landesjugendamt: Personalbemessung der Jugendämter in Bayern (PeB). Projektbericht und Handbuch. München 2009 [korrekt: 2010].

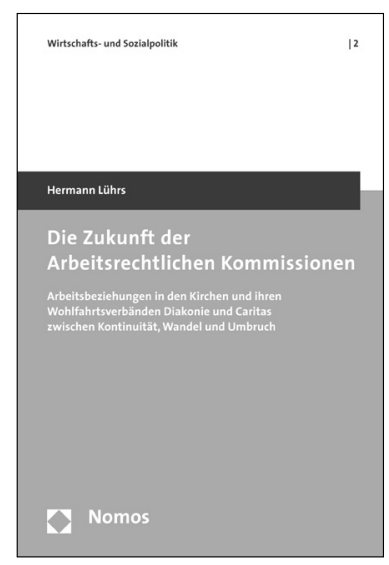

\section{Die Zukunft der Arbeits- rechtlichen Kommissionen}

Arbeitsbeziehungen in den Kirchen und ihren Wohlfahrtsverbänden Diakonie und Caritas zwischen Kontinuität, Wandel und Umbruch

Von Dr. Hermann Lührs

2010, 279 S., brosch., 49,- $€$, ISBN 978-3-8329-5183-2

(Wirtschafts- und Sozialpolitik, Bd. 2)

Erstmals wird in dieser Studie das System der Arbeitsrechtlichen Kommissionen in den Kirchen, Diakonie und Caritas sozialwissenschaftlich untersucht. Geboten wird ein umfassender und detaillierter Überblick über Entstehung, Gegenwart und Zukunftsperspektiven der Arbeitgeber/Arbeitnehmerbeziehungen in diesem Feld. Die Weichenstellungen des arbeitspolitischen Sonderweges der Kirchen seit 1949 und dessen institutionelle Persistenz werden pfadtheoretisch herausgearbeitet. Im Querschnitt werden aktuelle Konflikttendenzen seit der Ablösung der kirchlichen Vertragsordnungen vom BAT im Jahr 2005 erhoben und die Faktoren des Wandels und eines möglichen Pfadabbruches dargelegt und bewertet.

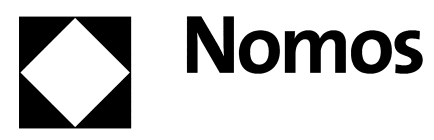

Bitte bestellen Sie im Buchhandel oder versandkostenfrei unter $\downarrow$ www.nomos-shop.de 